\title{
Temporal rarity is a better predictor of local extinction risk than spatial rarity
}

\author{
Peter A. Wilfahrt (D) 1,33 Ashley L. Asmus (DD, ${ }^{1}$ Eric W. Seabloom (DD 1 Jeremiah A. Henning (iD $, 1,2$ \\ Peter Adler (iD,${ }^{3}$ Carlos A. Arnillas (iD) ${ }^{4}$ Jonathan D. Bakker (iD) ${ }^{5}$ Lori Biederman (iD) ${ }^{6}$

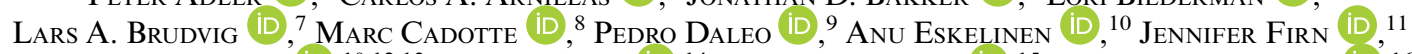 \\ W. Stanley Harpole (iD, ${ }^{10,12,13}$ Yann Hautier (iD, ${ }^{14}$ Kevin P. Kirkman (iD) ${ }^{15}$ Kimberly J. Komatsu (iD) 16 \\ Ramesh Laungani, ${ }^{17}$ Andrew MacDougall, ${ }^{18}$ Rebecca L. McCulley (iD, ${ }^{19}$ Joslin L. Moore, ${ }^{20}$ John W. \\ Morgan, ${ }^{21}$ Brent Mortensen (iD), ${ }^{22}$ Raul Ochoa Hueso (iD, ${ }^{23}$ Timothy Ohlert (iD, ${ }^{24}$ Sally A. Power (iD, 25 \\ Jodi Price (iD), ${ }^{26}$ Anita C. Risch iD, ${ }^{27}$ Martin Schuetz, ${ }^{27}$ Lauren Shoemaker (iD), ${ }^{28}$ Carly Stevens (iD), 29 \\ Alexander T. Strauss (iD, ${ }^{1,30}$ Pedro M. Tognetti, ${ }^{31}$ Risto Virtanen (iD,${ }^{32}$ and Elizabeth T. Borer (iD) 1 \\ ${ }^{1}$ Department of Ecology, Evolution, and Behavior, University of Minnesota, St. Paul, Minnesota 55108 USA \\ ${ }^{2}$ Department of Biology, University of South Alabama, Mobile, Alabama 36688 USA \\ ${ }^{3}$ Department of Wildland Resources and the Ecology Center, Utah State University, Logan, Utah 84322 USA \\ ${ }^{4}$ Department of Physical and Environmental Sciences, University of Toronto Scarborough, Toronto, Ontario M1C 1 A4 Canada \\ ${ }^{5}$ School of Environmental and Forest Sciences, University of Washington, Seattle, Washington 98195 USA \\ ${ }^{6}$ Department of Ecology, Evolution, and Organismal Biology, Iowa State University, Ames, Iowa 50011 USA \\ ${ }^{7}$ Department of Plant Biology and Program in Ecology, Evolutionary Biology and Behavior, Michigan State University, East Lansing, \\ Michigan 48824 USA \\ ${ }^{8}$ Department of Biological Sciences, University of Toronto Scarborough, Toronto, Ontario M1C 1 A4 Canada \\ ${ }^{9}$ Instituto de Investigaciones Marinas y Costeras (IIMYC), CONICET - UNMDP, Mar del Plata, Argentina \\ ${ }^{10}$ Department of Biology, German Centre for Integrative Biodiversity Research (iDiv), Leipzig 04103 Germany \\ ${ }^{11}$ School of Biology \& Environmental Science, Queensland University of Technology, Brisbane, Queensland 4000 Australia \\ ${ }^{12}$ Department of Physiological Diversity, Helmholtz Center for Environmental Research - UFZ, Permoserstrasse 15, Leipzig 04318 \\ Germany \\ ${ }^{13}$ Martin Luther University Halle-Wittenberg, am Kirchtor 1, Halle (Saale) 06108 Germany \\ ${ }^{14}$ Ecology and Biodiversity Group, Department of Biology, Utrecht University, Utrecht 3584 The Netherlands \\ ${ }^{15}$ School of Life Sciences, University of KwaZulu-Natal, Scottsville 3209 South Africa \\ ${ }^{16}$ Smithsonian Environmental Research Center, Edgewater, Maryland 21037 USA \\ ${ }^{17}$ Department of Biology, Doane University, Crete, Nebraska 68333 USA \\ ${ }^{18}$ Department of Integrative Biology, University of Guelph, Guelph, Ontario N1G 2 W1 Canada \\ ${ }^{19}$ Department of Plant \& Soil Sciences, University of Kentucky, Lexington, Kentucky 40546 USA \\ ${ }^{20}$ School of Biological Sciences, Monash University, Clayton, Victoria 3800 Australia \\ ${ }^{21}$ Department of Ecology, Environment \& Evolution, La Trobe University, Bundoora, Victoria 3086 Australia \\ ${ }^{22}$ Department of Biology, Benedictine College, Atchison, Kansas 66002 USA \\ ${ }^{23}$ Department of Biology, University of Cádiz, Cádiz 11001 Spain \\ ${ }^{24}$ Department of Biology, University of New Mexico, Albuquerque, New Mexico 87131 USA \\ ${ }^{25}$ Hawkesbury Institute for the Environment, University of Western Sydney, Penrith, New South Wales 2751 Australia \\ ${ }^{26}$ Institute of Land, Water and Society, Charles Sturt University, Albury, New South Wales 2678 Australia \\ ${ }^{27}$ Swiss Federal Institute for Forest, Snow and Landscape Research WSL, Birmensdorf 8903 Switzerland \\ ${ }^{28}$ Botany Department, University of Wyoming, Laramie, Wyoming 82071 USA \\ ${ }^{29}$ Lancaster Environment Center, Lancaster University, Lancaster LA1 4 YQ UK \\ ${ }^{30}$ Odum School of Ecology, University of Georgia, Athens, Georgia 30602 USA \\ ${ }^{31}$ IFEVA, Facultad de Agronomía, Universidad de Buenos Aires, CONICET, Buenos Aires, Argentina \\ ${ }^{32}$ Department of Biology, University of Oulu, Oulu 90570 Finland
}

Citation: Wilfahrt, P. A., A. L. Asmus, E. W. Seabloom, J. A. Henning, P. Adler, C. A. Arnillas, J. D. Bakker, L. Biederman, L. A. Brudvig, M. Cadotte, P. Daleo, A. Eskelinen, J. Firn, W. S. Harpole, Y. Hautier, K. P. Kirkman, K. J. Komatsu, R. Laungani, A. MacDougall, R. L. McCulley, J. L. Moore, J. W. Morgan, B. Mortensen, R. Ochoa Hueso, T. Ohlert, S. A. Power, J. Price, A. C. Risch, M. Schuetz, L. Shoemaker, C. Stevens, A. T. Strauss, P. M. Tognetti, R. Virtanen, and E. T. Borer. 2021. Temporal rarity is a better predictor of local extinction risk than spatial rarity. Ecology 102(11):e03504. 10.1002/ecy.3504

Abstract. Spatial rarity is often used to predict extinction risk, but rarity can also occur temporally. Perhaps more relevant in the context of global change is whether a species is core to a community (persistent) or transient (intermittently present), with transient species often susceptible to human activities that reduce niche space. Using 5-12 yr of data on 1,447 plant species from 49 grasslands on five continents, we show that local abundance and species persistence under ambient conditions are both effective predictors of local extinction risk following

Manuscript received 4 January 2021; revised 4 May 2021; accepted 3 June 2021. Corresponding Editor: Kathryn L. Cottingham.

${ }^{33}$ E-mail: pawilfahrt@gmail.com 
experimental exclusion of grazers or addition of nutrients; persistence was a more powerful predictor than local abundance. While perturbations increased the risk of exclusion for low persistence and abundance species, transient but abundant species were also highly likely to be excluded from a perturbed plot relative to ambient conditions. Moreover, low persistence and low abundance species that were not excluded from perturbed plots tended to have a modest increase in abundance following perturbance. Last, even core species with high abundances had large decreases in persistence and increased losses in perturbed plots, threatening the longterm stability of these grasslands. Our results demonstrate that expanding the concept of rarity to include temporal dynamics, in addition to local abundance, more effectively predicts extinction risk in response to environmental change than either rarity axis predicts alone.

Key words: core-transient; extinction risk; grasslands; herbivores; NutNet; nutrients; rarity.

\section{INTRODUCTION}

Humans are changing many environmental conditions concurrently including climate, nutrient supply rates, and disturbance regimes (Steffen et al. 2015), and plant communities respond to these changes with shifts in diversity, distribution, and composition (Franklin et al. 2016). However, determining which species will be adversely affected by global change is challenging because shifts in abundance and extinctions arise not only from altered environmental conditions, but also from stochastic events (Shoemaker et al. 2020). Rare species are generally assumed to be more sensitive to human perturbations than abundant, common, or broadly distributed species (Gaston 1994, Manne and Pimm 2001, Dawson et al. 2012, Duwyn and MacDougall 2015). The sensitivity of rare species is often attributed to having a narrower niche, more conservative resource acquisition, or a slower growth rate relative to their neighbors (Grime 1977, Rabinowitz 1981, Rabinowitz et al. 1984, Reich et al. 1999). Rare species also may be more likely to become extinct because a restricted geographic range or small population size can intensify the effects of demographic stochasticity on fluctuations in population sizes (Lande 1993). While rare species have long been defined as those having low relative abundance within communities, infrequent occurrence among communities, or a restricted geographic range (Rabinowitz 1981, Hanski 1982), these spatially explicit definitions of rarity neglect a potentially important temporal component of rarity.

Monitoring species occupancy in a patch through time allows a temporal axis of rarity to emerge, complementary to spatial metrics of rarity. By explicitly characterizing the temporal axis, communities can be separated into persistent "core" species that are present most of the time, and "transient" species that are intermittently present (Magurran and Henderson 2003, Ulrich and Ollik 2004, Shade and Gilbert 2015). The core-transient framework provides a temporal analog to the coresatellite hypothesis (Hanski 1982), which arrayed species on a rarity axis (i.e., core to satellite) in terms of abundance and patch occupancy. While an implicit temporal component of the core-satellite framework is possible, where species may switch core-satellite designations through time (Collins and Glenn 1991), recent studies demonstrate that transient, temporally rare species are ecologically distinct from satellite, spatially rare species (Supp et al. 2015). For instance, a satellite species in a region could be both persistent and abundant (e.g., temporally core) at a single site and a regionally core species could be locally transient at the margins of its range. Core and transient species have different life histories, where transient species tend to be smaller (Dolan et al. 2009), have shorter generation times, persist by seedbanking or dispersal (Supp et al. 2015), and may lack strong trait-environmental linkages (Umaña et al. 2017). In contrast, core species tend to be more strongly tied to their local environment (Umaña et al. 2017) and governed by species interactions (Magurran and Henderson 2003, Coyle et al. 2013). Based on this generality, core and transient species should also respond differently to environmental perturbation (Coyle et al. 2013, Supp et al. 2015) as perturbations can reduce environmental suitability for core species while creating colonization opportunities for transients, as novel conditions may arise from the perturbation that can be exploited (Roxburgh et al. 2004).

The core-transient framework generally proceeds from the assumption that persistence and abundance are positively correlated (Magurran and Henderson 2003), but there are many exceptions to this rule that depend on the underlying dynamics of perturbation. For example, some transient species track resources through time and space (Levin et al. 1984, Supp et al. 2015), emerging from the seedbank and becoming dominant in the community for a short time after a disturbance or resource pulse (Shade et al. 2014). At the opposite end of the rarity spectrum are resource specialists that persist at low abundance, either because they are limited by their competitive ability relative to other members of their community, or because they thrive in a narrow, stable niche (Grime 1977, Rabinowitz 1981). While transient species may rapidly exploit new environmental conditions, such species should experience the most detrimental effects of homogenizing perturbations that reduce niche dimensionality and, in turn, lead to stronger resource competition (Mouillot et al. 2013, Harpole et al. 2016). In sum, the competitive strategies of species can differ according to whether a species persists or briefly occurs at low or high relative abundances in a community. Thus, recasting species rarity into temporal (core to transient continuum) and spatial 
(locally abundant to locally scarce continuum) axes of rarity capture important life-history differences that may influence a species' response to environmental perturbations, including the likelihood of local extinction.

Here, we build from the core-transient framework by explicitly treating abundance and persistence as complementary predictors of species' responses to environmental perturbations (Fig. 1). We explore how grassland species with different temporal and spatial rarity attributes respond to two widespread environmental perturbations, nutrient addition and vertebrate herbivore exclusion. Previous studies have found that both spatial and temporal turnover in species composition in unfertilized plots are at least as important as the effects of nutrient addition in driving community responses (e.g., biomass production; Hillebrand et al. 2017, Hodapp et al. 2018, Langley et al. 2018), demonstrating the importance of quantifying both species' abundance and persistence under ambient conditions. Nutrient addition and herbivore exclusion tend to reduce grassland plant species diversity (Borer et al. 2014b, Harpole et al. 2016), with species responding in a non-random fashion. Species with traits such as rapid growth increase in abundance more rapidly than slow-growing species when herbivores are removed and nutrients are added (Lind et al. 2013), and exotic species increase in abundance more rapidly than natives (Seabloom et al. 2015), again suggesting the likelihood of unique spatial and temporal responses to environmental change.

We used plant community data from 49 grasslands on five continents, with each site participating in a longterm (5-12 yr at the time of this study), globally

A

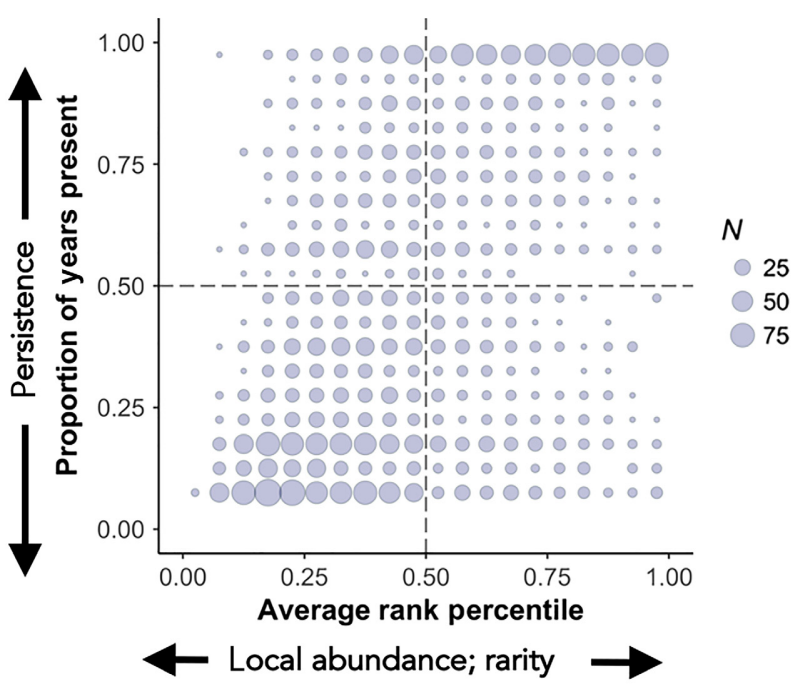

distributed nutrient addition and herbivore exclusion experiment (Borer et al. 2014a). This allowed us to calculate the average abundance and persistence of each species, and to examine how these temporal and spatial attributes predicted responses to environmental perturbation. First, we examined the degree to which abundance and persistence were correlated in control plots and the association of abundance and persistence with species functional types (i.e., life span and lifeform). Second, we examined how nutrient addition and herbivore exclusion altered species' abundance and persistence dynamics. Third, we examined how a species' abundance and persistence in control plots predicted species' exclusion from a plot due to nutrient addition and herbivore exclusion. Fourth, we determined the extent to which species' abundance and persistence jointly predicted changes in spatial and temporal rarity dynamics and species loss by assigning them to discrete abundance and persistence categories (Fig. 1) and examining the likelihood of categorical switches or exclusion following perturbation. Our work demonstrates that distinguishing spatial and temporal rarity comprises a general framework for predicting species responses to environmental change that relies on basic abundance data and is applicable across ecosystems.

\section{Methods}

\section{Nutrient Network experiment}

We used plant species' relative abundance data from The Nutrient Network (NutNet) experiment (Borer

B

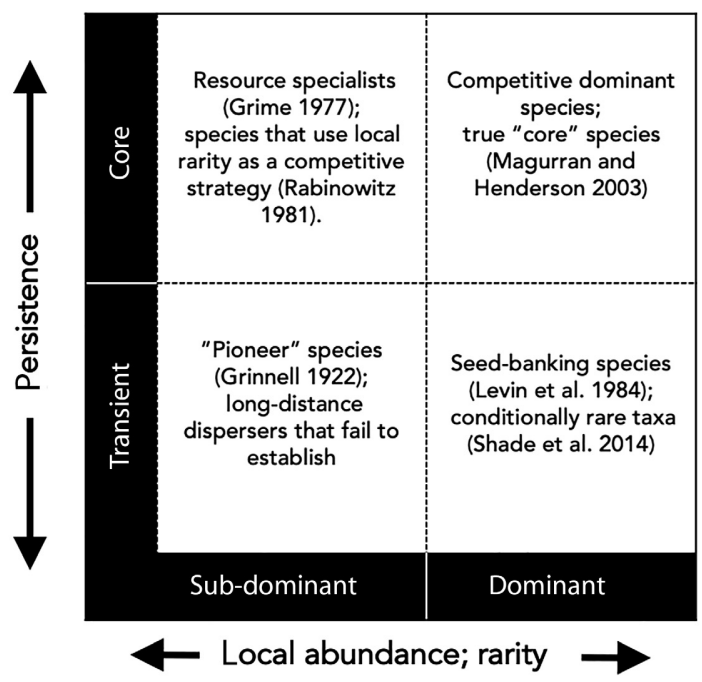

FIG. 1. Two views of persistence and abundance employed in this study. In (A), a continuous-axis approach for evaluating species' abundance (rank percentile abundance, i.e., the relative abundance of species in a plot); and persistence (the proportion of years a species is present in a plot). Data shown are all species observations from control plots at all 50 sites included in the study; observations are binned to the nearest 5\% for clarity, with the size of each dot indicating the number $(N)$ of observations in each bin. In (B), species are placed into categories that correspond to these demographic qualities, building upon the core-transient framework (Magurran and Henderson 2003). Some examples of each category are provided. 
et al. 2014a, 2017). Data were retrieved from the NutNet database on 2 August 2019. We selected data from sites that had 5-12 yr of data, in total, 49 sites (Appendix S1: Fig. S1, Table S1). Standardized treatments were applied in $5 \times 5 \mathrm{~m}$ plots at every site: nutrient addition (nitrogen, phosphorus, potassium each added at $10 \mathrm{~g} \cdot \mathrm{m}^{-2} \cdot \mathrm{yr}^{-1}$, and $100 \mathrm{~g} / \mathrm{m}^{2}$ of a standardized micronutrient mixture added in the first year), herbivore exclusion (fences installed to exclude vertebrate herbivores $>50 \mathrm{~g}$ ), nutrient addition combined with herbivore exclusion, and controls. At each site, treatment plots were nested within blocks in a fully randomized design, with each site replicating the experiment in at least three, and up to six, plots. Of the 49 sites included in the analysis, all implemented controls and the nutrient addition treatment, and 43 sites implemented the herbivore exclusion and combined herbivore exclusion and nutrient addition treatments. In each year, including the pretreatment year, percent cover of each species was visually estimated to the nearest percent in a permanent $1 \times 1 \mathrm{~m}$ quadrat within each $5 \times 5 \mathrm{~m}$ plot. In total, our data set contained 55,226 species-cover observations of 1,592 unique taxa, all of which were identified to genus, and 1,447 of which were identified to species. Perennial graminoids accounted for $49.5 \%$ of relative cover across all sites and all years, though 14 of the 49 sites were more herb dominated than graminoid dominated.

\section{Statistical analysis}

Deriving measures of persistence and abundance.-To standardize across sites that differed in the length of time they had been monitored, we used the proportion of years a species was observed in a plot as our measure of persistence (Appendix S1: Fig. S2). Following similar studies of plant extinction risk that rely on percent cover data (e.g., Suding et al. 2005, Collins et al. 2008), we used rank percentiles as our measure of species' abundance, averaged across all years where a species was present in a plot. Using rank percentiles standardized percent cover data across sites and years that differed in species richness and provided a direct measure of a species' relative abundance in a community. For example, a rank percentile of 0.75 corresponds to a species being more abundant than three quarters of its neighbors.

Assigning species to persistence-abundance categories.Although our analyses primarily treated persistence and abundance as continuous measures, we also assigned each species in each plot to one of four categories: core-scarce, core-abundant, transient-scarce, and transient-abundant (Fig. 1). "Core" species were present at least $50 \%$ of the time, while "transient" species were present less than half of the time; "abundant" species had a mean rank percentile greater than or equal to 0.5 (on average, more abundant than half of the species in the plot when the species was present, zeroes excluded), while "scarce" species' rank percentile was less than 0.5 . Such cutoff values are necessarily arbitrary as there is no standard way to separate core and transient species (Coyle et al. 2013), but we conducted additional analyses by assessing thresholds of 0.33 and 0.66 to understand how this choice affected our results. Analyses that evaluated species' exclusion rates in response to experimental treatments included one additional category, "absent": these were species that were not observed in a particular plot but were found in one or more other plots at a site. We use the term exclusion to indicate either failure to persist or failure to establish but cannot distinguish between these two mechanisms. In this study, we use these categorical analyses to understand how species' abundance and persistence interact to affect species and rely on our continuous abundance and persistence axes to more accurately understand abundance and persistence as independent drivers.

Relationships between species' persistence, average abundance and traits under ambient conditions. - To establish a baseline for our experimental treatment effects, we used data from control plots to conduct an exploratory analysis of the relationships between persistence, abundance, and species' traits, under ambient conditions. First, we quantified the degree to which a species' abundance predicted its persistence. This analysis relied on a simple linear mixedeffects model where persistence (proportion of years present) was the response variable, average abundance for the years where the species was present was the predictor variable, and site was included as both a random slope and intercept. To quantify the strength of the relationship between persistence and abundance, we calculated the marginal $R_{\text {GLMM }}^{2}$, the amount of variance explained by the fixed effects (i.e., abundance) in the mixed-effects model (Nakagawa et al. 2017). While our persistence and abundance indices are insensitive to species richness, sites that have more species per plot will nonetheless be more heavily weighted in analyses by providing more data points. We tested for any possible effect of site richness in this baseline analysis in two ways. First, we examined whether the observed relationship between persistence and abundance was influenced by more species rich sites by using linear models to test whether site richness predicted either the random site intercept or the random site slope estimates extracted from the linear mixed-effect model. Second, we tested if including site richness as a fixed effect had a significant effect or improved model AIC. We used this latter approach to test for site richness effects in all models described from here on.

Next, we evaluated whether persistence or abundance differed according to two sets of trait-based categorical schema commonly used in grassland studies: morphological grouping (graminoids, legumes, forbs, and woody taxa) and life span (annual and biennial vs. perennial and indeterminate life spans). We created two separate generalized linear mixed-effects models wherein morphological grouping, life span and their interaction were predictors of either persistence or average abundance, and site was a random intercept. We used a binomial 
link function for these models as response variables were non-normally distributed but bounded between 0 and 1 .

Finally, we explored the correspondence between the two trait-based schema and our persistence-abundance classifications using a multinomial logistic model, a type of logistic regression that allows more than two outcomes (Ripley and Venables 2016). Using a categorical approach allowed us to explore the way species' characteristics mapped to persistence and abundance simultaneously, rather than separately. In this model, morphological group, life span and their interaction were included as predictors of species' persistence-abundance category; site was included as an additional main effect as random effects were not supported in this type of model.

Evaluating whether nutrient addition and herbivore exclusion affect species differently according to species' placement along the core-transient continuum. - We next determined if a species' abundance and persistence under ambient conditions (Appendix S1: Fig. S2) predicted its abundance and persistence under altered conditions. We compared the abundance and persistence of species in control plots to the abundance and persistence of the same species in other control plots at the same site (to account for ambient variability in species' demography) and in treatment plots at the same site. These comparisons restricted us to the subset of species that were present in at least one control plot and one other plot ( $70 \%$ of all species in the data set).

Using this data set of within-species, across-plot comparisons of species abundance and persistence qualities, we specified linear mixed-effects models with species' abundance and persistence in each control plot as predictors of the same species' abundance and persistence in all other control and treatment plots at the same site. Both of these predictors also were allowed to interact with nutrient addition and herbivore exclusion including threeway interactions (i.e., persistence $\times$ nutrients $\times$ herbivore exclusion; abundance $\times$ nutrients $\times$ herbivore exclusion). We created a species-plot ID term and nested it within site as a random intercept in this model to account for the multiple comparisons made from a single species within each control plot. Thus, the coding in $\mathrm{R}$ following the lme4 package nomenclature was as follows:

$$
\begin{aligned}
& \text { persistence } \text { comparison }_{\text {persistence }} \text { control } \\
& \quad \times \text { hutrients } \\
& \times \text { herbivore exclusion }+ \text { abundance } \\
& \quad \times \text { nutrientrol } \\
& \quad+(1 \mid \text { site } / \text { speciesivore exclusion } \\
&
\end{aligned}
$$

$$
\begin{aligned}
& \text { abundance }_{\text {comparison }} \sim \text { persistence }_{\text {control }} \times \text { nutrients } \\
& \times \text { herbivore exclusion }+ \text { abundance }_{\text {control }} \\
& \times \text { nutrients } \times \text { herbivore exclusion } \\
& +(1 \mid \text { site } / \text { speciesID })
\end{aligned}
$$

These models allowed us to determine (1) how abundance and persistence in ambient conditions predicted abundance and persistence following environmental perturbations and (2) to quantify the ways in which treatments acted on species differently depending on their abundance and persistence. We additionally examined a full model that added species' life span and lifeform along with abundance and persistence axes, all interacting with nutrient addition and herbivore exclusion as predictors, and a model with only life span and lifeform interacting with nutrient addition and herbivore exclusion, to assess whether these categorical traits added unique information to abundance and persistence axes.

\section{Evaluating the degree to which abundance and persistence predict local species loss}

To understand how abundance and persistence predicted species loss following nutrient addition and herbivore exclusion, we evaluated whether species present at the site scale existed within a plot anytime in the last five years of study (Appendix S1: Fig. S3). Species that were absent in all years were recorded as zero, representing an absence that could be attributed to local extinction or a failure to establish. Similar to the previous analysis, we assigned each species in a plot a presence/absence score $(1,0)$ from the final five years of study and an abundance and persistence score from control plots across all years of data collection for each site. We conducted linear mixed-effect models predicting a species' presence/ absence by abundance and persistence in controls, each interacting with nutrient addition and herbivore exclusion, a binomial link function, and species-Plot-ID nested within site as random effects (Appendix S1: Fig. $\mathrm{S} 3$ ). The $\mathrm{R}$ coding following the lme4 package nomenclature was as follows:

$$
\begin{aligned}
& \text { presence }_{\text {comparison }} \sim \text { persistence } \\
& \quad \times \text { herbivivol } \\
& \times \text { nutrients } \\
& \times \text { nutrients } \times \text { herbivore exclusion } \\
& +(1 \mid \text { site } / \text { taxonID })
\end{aligned}
$$

Because failure to establish is not necessarily due to competitive exclusion or herbivory, and persistence and absence are not independent, establishing baseline relationships in the control plots allowed us to contextualize the effects of nutrient addition and herbivore exclusion.

Evaluating whether environmental perturbations switched species from their baseline core-transient, abundant-scarce categories. - Our analyses of treatment effects (until now) treated abundance and persistence independently. In order to understand how they jointly impacted changes, we used our categorical assignment methodology from above (i.e., core or transient and locally abundant or scarce). This model specifically evaluated (1) whether treatments created opportunities for species to switch from one persistence-abundance category to another and (2) if treatments altered species' absences based on 
abundance and persistence, again measured as the probability that a species with a given category in a control plot would be absent in a treatment plot for the final five years of study. To do this, we first assigned species to persistence-abundance categories for the final five-year interval at their site. We then constructed a data set of across-plot, within-site, within-species comparisons. We omitted pretreatment years from the final five-year intervals, leaving six sites with only four years of data for this analysis. We applied a multinomial logistic model to this data set, evaluating the degree of correspondence between species' persistence-abundance category assignments across control plots (to capture ambient variability in species' categorical assignment) and between control and treatment plots. The $\mathrm{R}$ coding was as follows:

$$
\begin{aligned}
& \text { category }_{\text {comparison }} \sim \text { category }_{\text {control }} \times \text { nutrients } \\
& \times \text { herbivore exclusion }+ \text { site }
\end{aligned}
$$

Because some sites had longer term data, and therefore an increased length of exposure to treatments, we split sites into early $(5-8 \mathrm{yr})$ and late $(9-12 \mathrm{yr})$ to see if effects were stronger in the later years. Additionally, we tested the sensitivity to the 0.5 cutoff for core-transient and abundant-scarce by shifting it to 0.33 and 0.66 and rerunning the analysis. We ran a final analysis that omitted any species in a control plot that had a persistence or abundance value between 0.33 and 0.66 to see whether the more extreme species reacted differently.

We conducted all statistical analyses in $\mathrm{R}$ version 4.0.2 ( $\mathrm{R}$ Core Team 2020). We used the $\mathrm{R}$ packages nnet for multinomial logistic models (Ripley and Venables 2016), lme4 for mixed-effects models (Bates et al. 2014), ImerTest for Wald tests of significance of mixed-effects model terms (Kuznetsova et al. 2017), and emmeans to conduct post-hoc tests and extract least-squares means estimated treatment effects for plotting (Lenth et al. 2018).

\section{RESULTS}

\section{Species' persistence, abundance and life history traits under ambient conditions}

We found a positive correlation between the abundance and persistence of species $(r=0.51$, marginal $R_{\mathrm{GLMM}}^{2}=0.27$, Est $=0.75 \pm 0.03, P<0.001$; Fig. 1 ; Appendix S1: Fig. S4). Despite this positive correlation, there were many exceptions to the rule. Of the species present in a plot less than $25 \%$ of years, $23 \%$ were abundant (mean rank $>50 \%$ ); conversely, of the species that persisted at least $75 \%$ of years, $30 \%$ were locally scarce (mean rank $<50 \%$ ). These results were not sensitive to species richness: (1) there was no relationship between site richness and the estimated site-level random intercepts or slopes (Appendix S1: Fig. S5) and (2) when site richness was included as a main effect, it did not improve model fit for any model we tested.
The combination of plant species' functional group and life span was a significant predictor of persistence $\left(F_{2,3954}=5.7, P=0.003\right)$ and abundance $\left(F_{2,3883}=6.8\right.$, $P=0.001$, Appendix S1: Tables S2, S3). Perennial and annual graminoids tended to persist longer and be more abundant than annual forbs and annual legumes; perennial graminoids tended to be more persistent than all forbs and annual legumes and tended to be more abundant than all forbs and legumes (Fig. 2A, B). Differences in abundance and persistence (considered as continuous variables) were also apparent in the correspondence between the core-abundance categorical scheme and plant species' functional group assignments, and between the categorical scheme and life span (Fig. 2 C, Appendix S1: Table S4). Core-abundant species were most likely to be perennial graminoids and least likely to be annual forbs (Fig. 2C, upper right), while core-scarce species were most likely to be perennial forbs, although few differences were significant in this designation (Fig. 2C, upper left). Meanwhile, transient-scarce species were more likely to be annual forbs or annual legumes and least likely to be graminoids or woody species (Fig. 2C, lower left). Transient-abundant species were more likely to be perennial graminoids than forbs (Fig. 2C lower-right), although differences between plant species' functional groups and life spans within this category were weak overall.

\section{Treatment effects on species persistence (continuous-axis approach)}

After establishing a baseline of species' spatial and temporal rarity in relation to each other and across functional groups, we tested how these rarity attributes changed in response to nutrient addition and herbivore exclusion. In general, abundance, persistence, and their positive correlation were preserved across control plots (Fig. 3; Appendix S1: Tables S5, S6). Nutrient addition, herbivore exclusion and their combination reduced species' persistence, with nutrient addition having stronger effects than herbivore exclusion, and the combination of herbivore exclusion and nutrient addition having the most detrimental effects (Fig. 3A, B, Appendix S1: Table S5). These detrimental treatment effects on persistence acted most strongly on species with high ambient persistence (nutrients $\times$ herbivore exclusion $\times$ ambientpersistence: $P=0.007$, Appendix S1: Table S5), with herbivore exclusion and nutrient addition estimated to decrease persistence 4.2 times as much for species with high persistence (i.e., near 1) vs. those with low persistence (i.e., near 0). Additionally, the detrimental effects of nutrient addition on persistence were more pronounced in species with low ambient abundance (nutrients $\times$ ambient-abundance: $P=0.004$ ), with nutrient addition decreasing the persistence of low abundance species twice as much as high abundance species; herbivore exclusion did not interact with ambient abundance (nutrients $\times$ herbivore exclusion $\times$ ambient-abundance, 
A

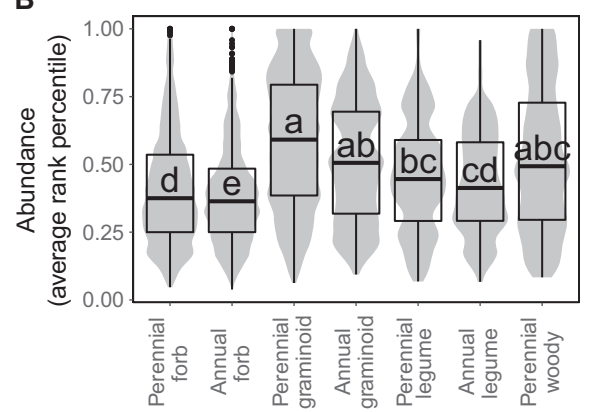

C

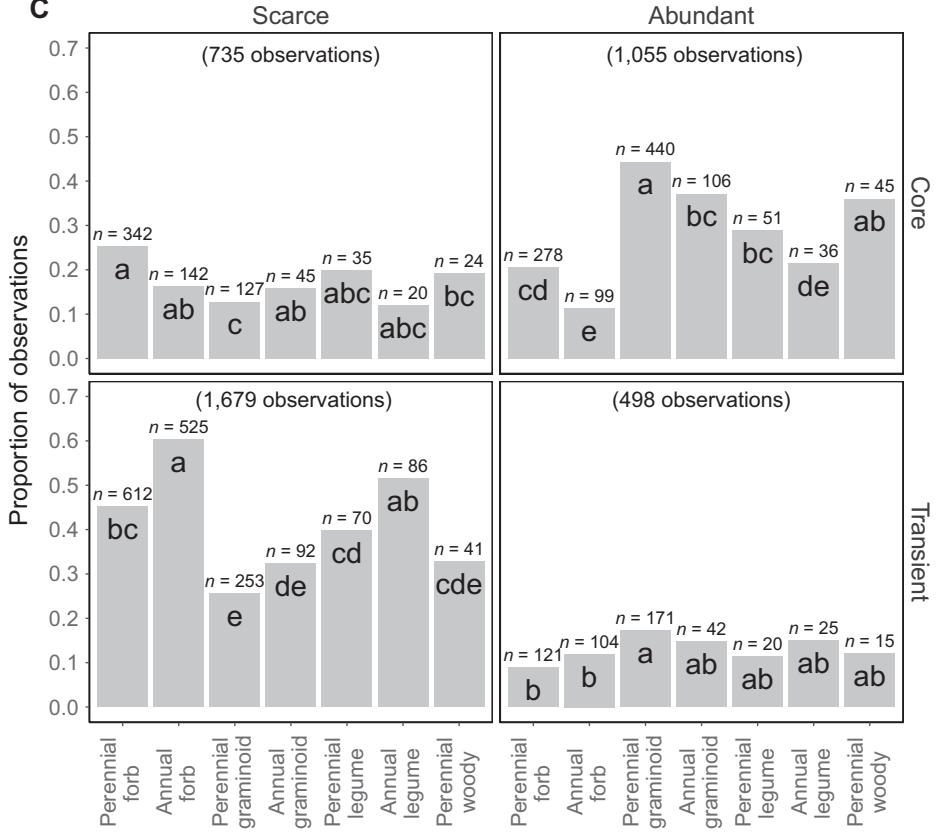

FIG. 2. Species' (A) persistence, (B) abundance, and (C) persistence-abundance signatures by plant functional group and life span for all control plots in the study. "Annual" species include biennials; "perennial" species include species with indeterminate life spans. Each observation is a species in a control plot monitored for 5-12 yr, depending on the length of study at each site. Lowercase letters denote significant differences in (A) persistence and (B) abundance, respectively, among plant functional types (leastsquares means estimates, Tukey HSD < 0.05). In (C), lower-case letters denote significant differences in the probability of category assignment among plant functional types (multinomial model contrasts, Tukey HSD $<0.05$ ).

$P=0.73 ;$ herbivore exclusion $\times$ ambient-abundance, $P=0.79$ ). Species abundance was conditionally influenced by nutrient addition based on species' abundance and persistence in control plots (nutrients $\times$ ambientpersistence, $P<0.001$; nutrients $\times$ ambient-abundance, $P=0.003$; Fig. 3C, D, Appendix S1: Table S6). Specifically, nutrient addition tended to increase the abundance of low persistence and low abundance species, while decreasing the abundance of high persistence and high abundance species. Herbivore exclusion showed a similar qualitative pattern, but only had a marginally significant interaction with ambient abundance $(P=0.08)$ and was otherwise insignificant (nutrients $\times$ herbivore exclusion $\times$ ambient-persistence, $P=0.50$; nutrients $\times$ herbivore exclusion $\times$ ambient-abundance, $P=0.39$ ). Effect sizes of abundance responses were much weaker than the observed persistence responses, which are directly comparable as they are both scaled from 0 to 1 . For instance, the largest change in estimated abundance following herbivore exclusion and nutrient addition was a decrease of 0.056 for high abundance species, or $34 \%$ of the largest estimated treatment effect on persistence. The largest increased abundance of 0.032 following perturbations at low baseline abundance and persistence was similarly small, only $22 \%$ of the largest absolute effect on persistence at low persistence and abundance. Including life span and lifeform slightly increased the predictive power of these models, though they were significantly less predictive on their own compared to the temporal and spatial rarity axes as predictors (Appendix S1: Table S7).

\section{Abundance and persistence and treatment effects on species loss}

We tested whether abundance and persistence interacted with treatment effects to drive local species loss. Higher persistence $(P<0.001$, Fig. 4A, Appendix S1: Table S8) and abundance in ambient plots $(P<0.001$, Fig. 4B, Appendix S1: Table S8) were associated with decreased species loss (in both ambient and treatment plots). The estimated increase in species loss from high to low persistence was 3.3 times higher than that from high to low abundance $(-0.729$ vs. -0.224$)$. Species loss increased with nutrient addition $(P<0.001)$ and herbivore exclusion $(P<0.001)$ relative to controls. Species loss changed unevenly with herbivore exclusion and nutrient addition along the persistence axis (nutrients $\times$ ambient-persistence, $P=0.037$ ). No significant interactions between treatments and abundance were detected (Appendix S1: Table S8), suggesting treatment effects on species loss were even along abundance and persistence gradients. However, the estimated increase in species loss from treatment effects was highest for low abundance species. Specifically, following herbivore exclusion and nutrient addition, low abundance species 

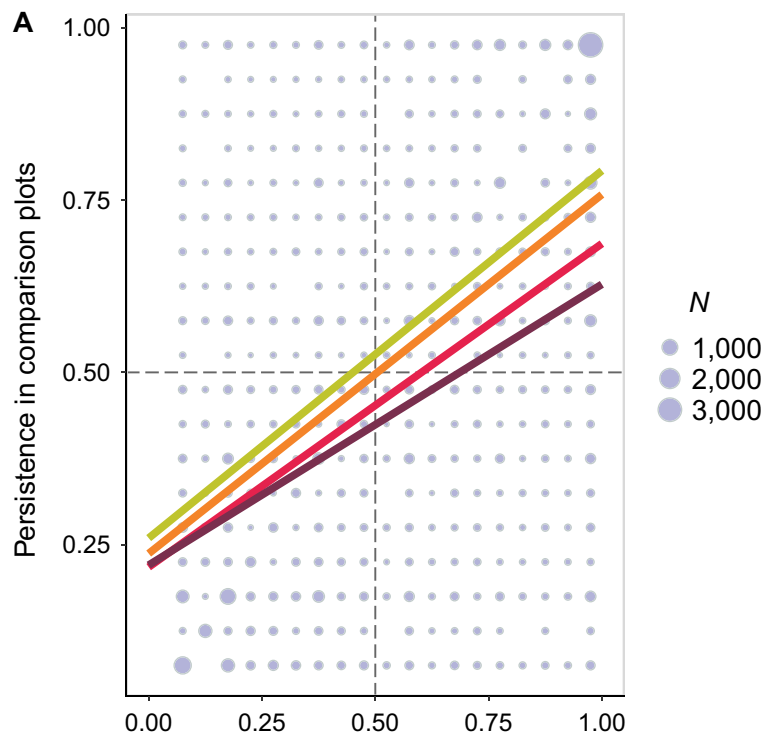

B

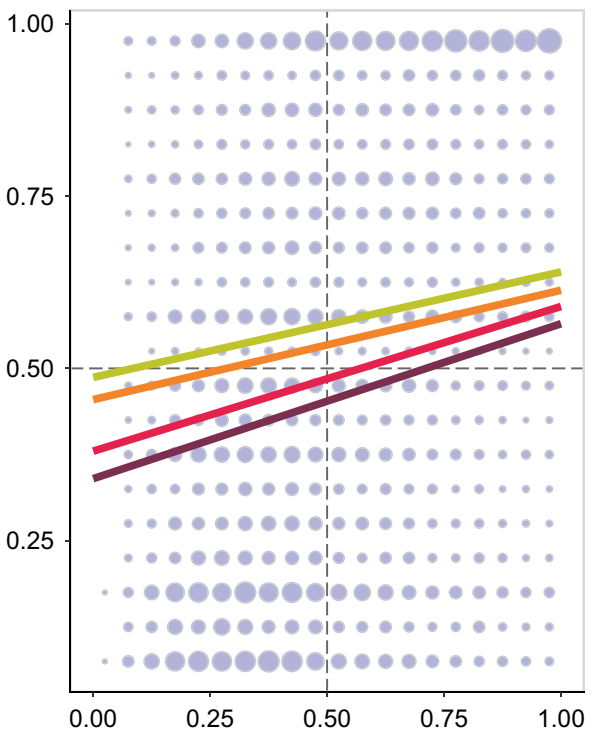

$N$

- 100

- 200

300

400

500
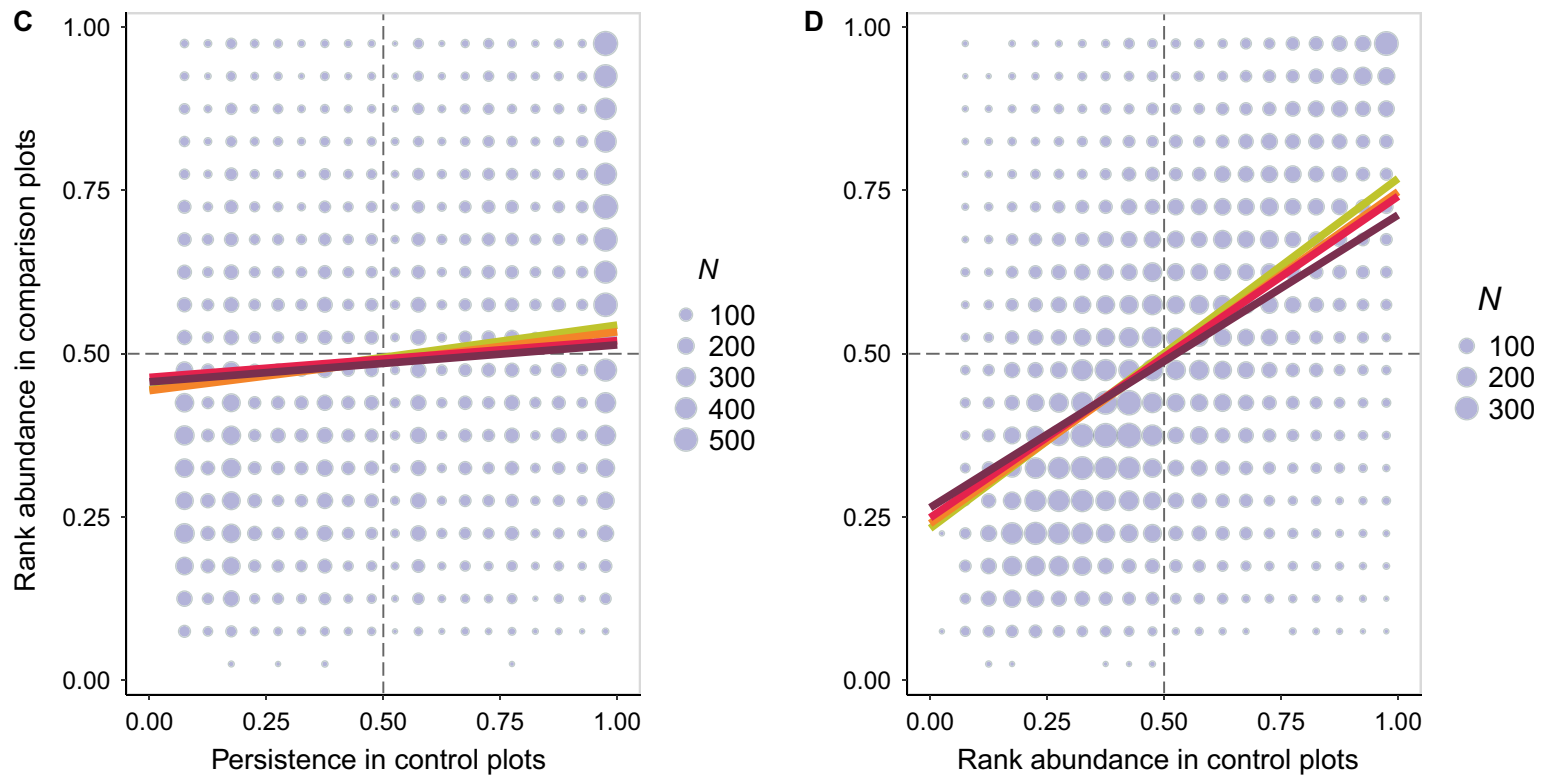

FIG. 3. Model estimates of a species' (A, C) persistence and (B, D) abundance in control plots predicting its (A, B) persistence and $(C, D)$ abundance in comparison plots. Persistence values of 1 indicate a species was found in a given plot every year of survey. Rank abundance values of 1 indicate a species was the most abundant species in a given plot. The green line shows baseline estimates of how control plots predict other control plots, while orange, red, and purple lines show treatment effects. Observations are binned to the nearest $5 \%$ for clarity, with the size of each dot indicating the number $(N)$ of observations in each bin.

had a $260 \%$ greater increase in loss than low persistent species relative to their baseline values and a $160 \%$ greater increase in losses than high abundance species relative to their baseline values. The marginal $R^{2}$ of the full model (AIC 50,456) with both abundance and persistence as predictors was 0.274 . If the persistence term and associated interactions were dropped (AIC 51,716), the marginal $R^{2}$ dropped to 0.123 , or more than a $55 \%$ decline in explanatory power. If the abundance term and interactions were dropped (AIC 50,511), the marginal $R^{2}$ dropped to 0.271 , less than a $1 \%$ decline in explanatory power. Both AIC and the marginal $R^{2}$ term indicate abundance was redundant when persistence was already considered, while persistence added unique information on species loss over abundance alone.

\section{Treatment effects on species' exclusion rates and categorical assignments}

Species with different categorical combinations of persistence and abundance reacted differently to nutrient addition and herbivore exclusion (Appendix S1: 

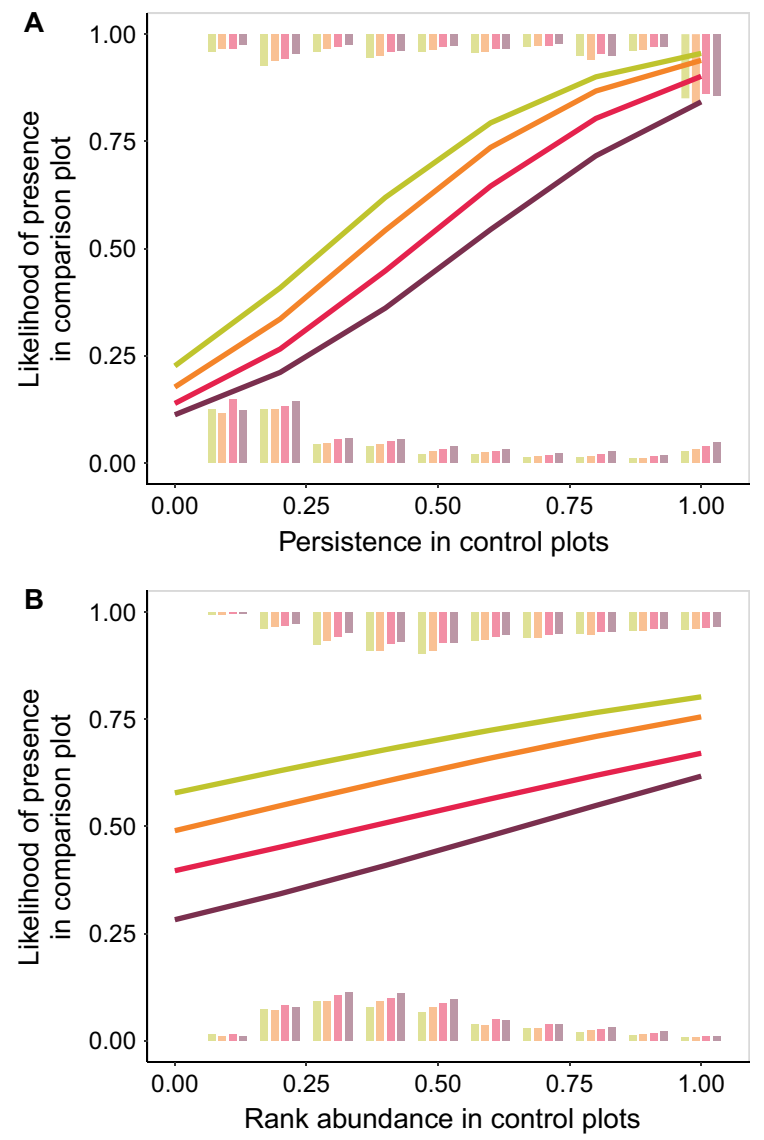

FIG. 4. Model estimates of species' exclusion from logistic regression mixed models showing the likelihood of a species being present (value of 1) in a comparison plot based on (A) persistence and (B) abundance in a control plot. Lower persistence, lower abundance, nutrient addition, and herbivore exclusion all increased the chance a species is excluded. No significant interactions were detected. Marginal histograms show the distribution of data within treatments (i.e., the absolute length of each bar for each color would add up to 1 in each plot), aggregated to the nearest 0.1 persistence/abundance value for clarity.

Table S9). Core-abundant species were the most stable category in control plots (Fig. 5A; Appendix S1: Table $\mathrm{S} 10$ ), retaining the same classification in the other control plots within the site $60 \%$ of the time. Species in all other categories were most likely to switch categories or be absent in other controls. Transient-abundant species were the least likely to be categorized the same way in another control plot and transient-scarce species were most likely to be absent in another plot.

Establishing the baseline tendency for species to shift categories allowed us to understand how nutrient addition and herbivore exclusion affected joint changes in species' abundance and persistence (Fig. 5B-D; Appendix S1: Table S10). In general, both treatments increased the likelihood that a species present in an ambient plot would be absent in a treatment plot, and this effect was stronger when both treatments were applied $(P<0.001$, Appendix S1: Table S9). While this was qualitatively clear in the previous analysis, the categorical switching analysis demonstrated several distinctions relating to species' joint abundance and persistence attributes. First, core-abundant species were most likely to decrease in stability (i.e., change categories) following perturbation, with up to $20 \%$ less core-abundant species remaining in this category in the presence of nutrient addition and herbivore exclusion; half of this decrease was attributable to increased absences and the other half was core-abundant species switching to the transientabundant or transient-scarce categorization. Second, transient-abundant species were 33\% more likely than core-scarce or transient-scarce species to become excluded in nutrient addition plots relative to other control plots. Increases in transient-abundant species' exclusion generally came at the expense of transient-abundant species shifting to other categories, while other categories' increased exclusion generally came at the expense of categorical stability (i.e., less likely to remain in the same category).

Because the length of study differed by site, we subdivided sites into "early" (5-8 yr) and "late" (9-12) to understand if effects differed through time. Effects in later years were generally stronger, but qualitatively similar (Appendix S1: Table S10). Significant effects of herbivore exclusion did not appear in early sites, suggesting species loss took longer to occur following fencing.

Given that 0.5 is an arbitrary cut-off for our categories, we tested additional thresholds for dividing species into categories (see Appendix S1: Table S11 for details). Lowering the threshold increased baseline absences of transient species and led to more equal increases in perturbance-induced absences in all categories. Increasing the threshold led to lower predicted rates of absences in all categories as all categories contained species with higher persistence and abundance scores, while absence increases from treatments remained high for transient species. Overall, this indicates that categorization is sensitive to the threshold we chose, yet it remained clear that even species with higher abundance scores were prone to exclusion following treatments if they were also transient.

\section{Discussion}

Abundance and persistence, while positively correlated, can affect species' responses to environmental perturbations in different ways. Nutrient addition and herbivore exclusion both reduced the persistence of species across the full gradient of species' baseline persistence and abundance, although the strongest reduction occurred in high persistence but low abundance species. These experimental perturbations affected species loss relatively evenly along persistence and abundance axes, but examining these rarity axes in tandem (i.e., with categorical assignments) revealed distinct responses. Species 
A

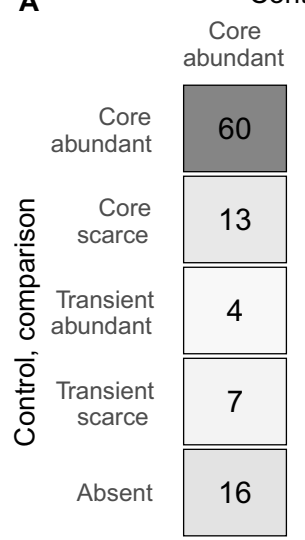

Co

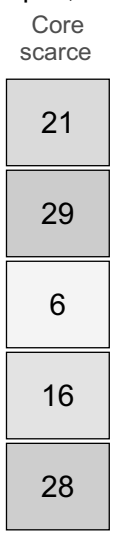

C

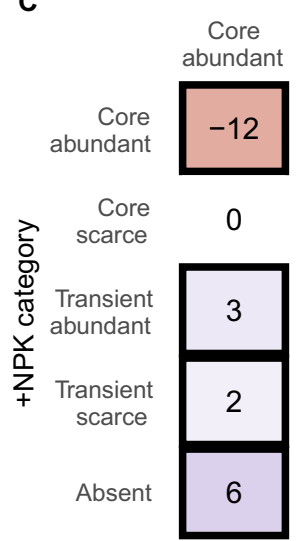

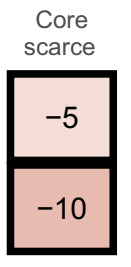

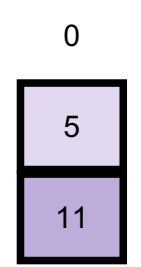

Transient

abundant Transient

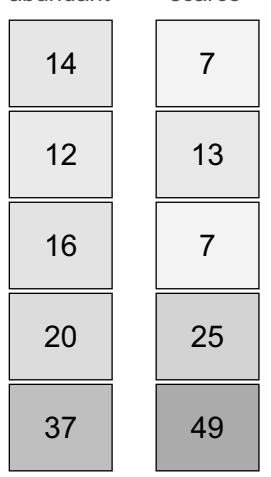

(ansient

Transient Transient abundant scarce
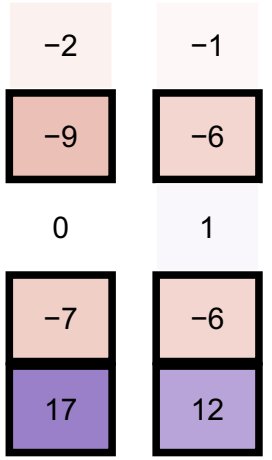

B

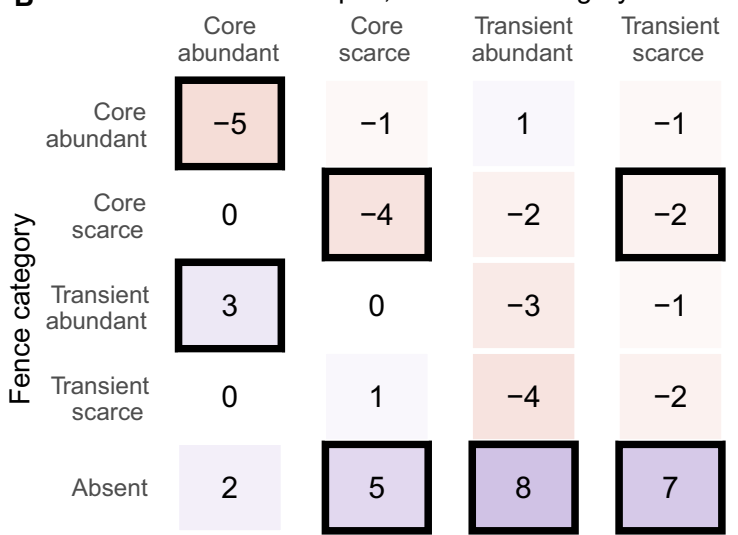

D

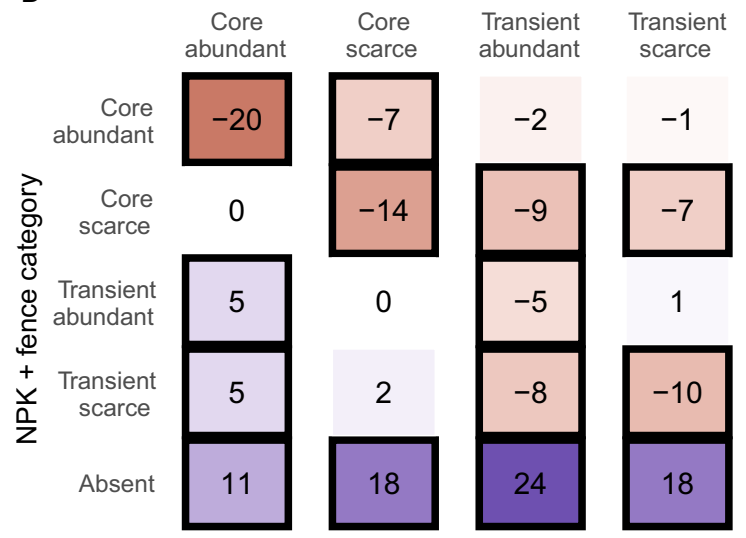

FIG. 5. (A) Categorical analysis of the ambient variability in plant species' persistence-abundance category assignment and (B-D) treatment effects on plant species' categorical assignments, estimated from a multinomial logistic model. In panel A, color density and numbers correspond to the percentage of species with a given designation in one control plot (column) that fall into the a given category in another control plot (row) at the same site. In panels B-D, color intensity and numbers correspond to treatment effects (rows) on the correspondence among categories. Treatment effect sizes are expressed as percent deviation from the values in panel A and heavy outlines indicate significant differences. Negative numbers with a red panel indicate a decreased probability of occurrence (shading is redundant with the number) and positive numbers with a blue panel indicate an increased probability of occurrence relative to the ambient panels A. See Appendix S1: Fig. S3 for a visual explanation of the analysis and Appendix S1: Table S10 for complete post hoc test results for each correspondence among categories.

that were transient-scarce under ambient conditions were most likely to be absent in another ambient community. Transient-abundant species had the largest increase in absences in perturbed communities. However, lowering the thresholds for categorizing species as transient or scarce shifted absence increases toward coreabundant and core-scarce species, suggesting that many of these absences were from species with moderately low persistence that persisted in ambient, but not perturbed, conditions. A handful of observational studies have described how temporally core and transient species are ecologically distinct (Coyle et al. 2013, Umaña et al. 2017), while others have focused on how spatial rarity can exacerbate species' extinction risk (Manne and Pimm 2001). By explicitly separating the spatial and temporal responses of species, our analysis of the NutNet experimental study provides evidence that temporally rare species in grasslands respond differently than locally scarce species to perturbations in both nutrient supply and herbivory.

Rather than working from the assumption that abundance and persistence are interchangeable (Magurran and Henderson 2003), our work treats these classifications as complementary species characteristics. Consistent with studies of other communities, we found that grasslands contain predominantly transient-scarce species, almost $50 \%$ more than the next most common category, core-abundant. Yet we also found species that were both transient and abundant, as well as those that persist year-after-year at low abundance, akin to those described by Rabinowitz et al. (1984). Our framework emphasizes the unique role that species with decoupled abundance and persistence can play in communities (Griggs 1940). Whereas spatially scarce and transient 
taxa are often excluded from community analyses (Shade et al. 2014, Shade and Gilbert 2015), our analyses were able to account for a sizable number of such species and suggest that they may play important roles in community dynamics under both ambient and altered conditions. In particular, species that have low persistence or abundance tended to be lost following perturbation, but those species that remained tended to have a small increase in abundance (considered as a continuous response). This suggests a suite of "evanescent" taxa (sensu Gleason 1926), potentially at a physiological disadvantage year to year, that can take advantage of windows of optimal environmental conditions. These taxa may serve as sentinels of longer-term biodiversity trends in response to environmental change (Shade et al. 2014). Meanwhile, dominant species are responsible for the bulk of ecosystem function in any given year (Grime 1998); the high instability of transient-abundant species, and their high rates of species loss in perturbed plots, suggests a role for core-transient dynamics in maintaining ecosystem function.

Our exploration of species' persistence and abundance under ambient conditions offers generalizable insights into the biology of temporally dynamic ecosystems, and grassland ecosystems in particular. We found that coreabundant species were relatively stable within sites. A species that was locally scarce in one plot, regardless of being core or transient, was most likely to be categorized as locally scarce in all other plots where present, while transient-abundant species fluctuated considerably in abundance and persistence among plots. In the current study, these categories arise from plant demographic characteristics and are closely related to plant species' life span (perennial vs. annual) and plant functional type (forb, graminoid). We observed that within a functional group, perennials were more likely to be core, whereas forbs were more likely to be scarce than graminoids. Even so, we found that many species fell outside their expected category. For example, $26 \%$ of annual forbs and $33 \%$ of annual legumes were core under ambient conditions. This suggests that the core and transient portions of communities comprise diverse species assemblages, and points to the possibility for niche complementarity in both space and time. Previous studies have demonstrated that species' functional type and life span effectively predict species' extinction risk (Suding et al. 2005) and richness loss due to nutrient addition (Tilman 1993). Similarly, we found that plant functional group and life span partially predicted treatment effects on species' dynamics. Our work builds from the earlier studies by demonstrating that incorporating species' placement along the core-transient continuum provided even greater predictive power of species loss, a metric that also generalizes beyond grassland systems and plant communities.

Background levels of species exclusion increased with species rarity, a finding we expected and is welldocumented in the literature, given that species with smaller populations are more sensitive to the effects of demographic stochasticity than abundant species (Matthies et al. 2004, Shoemaker et al. 2020). We also had expected to find that, after accounting for background rates of species loss, rare species would be more sensitive to treatment effects. Surprisingly, this was not always the case. While less abundant species had greater declines in persistence following perturbation, our results uncovered the surprising effect that decreases in persistence were largest for species with high persistence in ambient conditions. Additionally, species with the lowest persistence and abundance that were not excluded were more likely to slightly increase in abundance following perturbation. Because lower persistence and abundance were associated with more absences from other plots, this points to a pathway for perturbation to drive local extinction in core species at increased rates in the future. This prediction is partially supported by the result that core-abundant species were least likely to be excluded following perturbation but were most likely to switch categories (e.g., switch to transient or spatially rare) compared to species in other categories following perturbation. Moreover, transient-abundant species had the highest increases in exclusion due to perturbation. While this result was sensitive to the threshold for defining the categories, this effect is consistent with the idea that perturbations reduced resource gaps on which transient species rely, as supported by our continuous metric approach. Last, these effects generally became stronger through time. A recent meta-analysis suggests compositional changes and diversity loss are often delayed by more than 10 years after resource perturbations begin (Komatsu et al. 2019, Seabloom et al. 2020). Thus, the possibility emerges that while core-abundant species buffer extinction by being common in both space and time, these species tend to be strongly tied to their local environment (Umaña et al. 2017), and environmental changes that reduce the abundance or persistence of these core species may cause an outsized impact on their buffering effect.

Our findings on species' exclusion rates reflect not only extinction risk, but also the inability to colonize plots from year to year. Our study did not distinguish between loss of an established species vs. failure to establish within a plot, but more generally established a link between increased temporal and spatial rarity and risk of plot-level exclusion following experimental perturbations. These findings yield additional insight into related studies from this experiment. Harpole et al. (2016) found that nutrient addition reduces plant species diversity by simplifying the belowground niche, i.e., reducing the number and changing the relative importance of multiple limiting resources. A complementary study demonstrated that herbivore exclusion can magnify species losses due to nutrient addition if the aboveground plant tissue removed via herbivory decreases, intensifying competition for light (Borer et al. 2014b). Our study suggests that species with low persistence or 
abundance comprise a large proportion of these losses due to changes in nutrients or herbivory. Nonetheless, core species that are more tightly coupled to their environmental niche (Umaña et al. 2017), as well as abundant species that drive ecosystem function (Grime 1998), are also vulnerable. Earlier work demonstrated that larger species pools can buffer communities from species losses by allowing for greater turnover (Hodapp et al. 2018). We highlight that these buffering effects may be imperiled; even if total species number is buffered from some loss, perturbations like elevated nutrients can lead to greater community instability (Avolio et al. 2014, Hautier et al. 2014, 2015). In particular, decreases in the abundance and persistence of core-abundant species create communities that are more unpredictable, in ways that may be decoupled from changes to species richness (Hillebrand et al. 2017), and instability of local community diversity may destabilize larger scale diversity as well (Hautier et al. 2020).

In sum, our large-scale, long-term experimental work demonstrates that temporal and spatial stability are key, and unique, components of species rarity and, by explicitly quantifying each, we enhance our ability to assess extinction risk and community dynamics under environmental change. Although spatial rarity is more frequently assessed, our results demonstrate that temporal rarity provides a better predictor of extinction risk than spatial rarity. While we have focused on grasslands and their multi-year responses to experimental manipulations, we believe that this framework is generalizable to other systems, other types of perturbations, and over shorter or longer time scales.

\section{ACKNOWLEDGMENTS}

This work was generated using data from the Nutrient Network (http://www.nutnet.org) experiment, funded at the sitescale by individual researchers. Coordination and data management have been supported by funding to E. Borer and E. Seabloom from the National Science Foundation Research Coordination Network (NSF-DEB-1042132) and Long Term Ecological Research (NSF-DEB-1234162 and NSF-DEB1831944 to Cedar Creek LTER) programs, and the Institute on the Environment (DG-0001-13). A. Eskelinen was supported by the Academy of Finland (297191). We also thank the Minnesota Supercomputer Institute for hosting project data and the Institute on the Environment for hosting Network meetings. P. Wilfahrt and A. Asmus analyzed the data and wrote the paper; E. Seabloom, E. Borer, A. T. Strauss, and J. Henning helped frame the questions; J. Henning, A. T. Strauss, and J. D. Bakker contributed to analyses; all others contributed data and to writing. Full author contributions are listed in Appendix S1: Table S12.

\section{Literature Cited}

Avolio, M. L., S. E. Koerner, K. J. La Pierre, K. R. Wilcox, G. W. T. Wilson, M. D. Smith, and S. L. Collins. 2014. Changes in plant community composition, not diversity, during a decade of nitrogen and phosphorus additions drive aboveground productivity in a tallgrass prairie. Journal of Ecology 102:1649-1660.
Bates, D., M. Maechler, B. Bolker, and S. Walker. 2014. lme4: linear mixed-effects models using Eigen and S4. R package version 1.1-7. https://www.jstatsoft.org/article/view/v067i01

Borer, E. T., W. S. Harpole, P. B. Adler, E. M. Lind, J. L. Orrock, E. W. Seabloom, and M. D. Smith. 2014a. Finding generality in ecology: a model for globally distributed experiments. Methods in Ecology and Evolution 5:65-73.

Borer, E. T., et al. 2014b. Herbivores and nutrients control grassland plant diversity via light limitation. Nature 508:517520.

Borer, E. T., J. B. Grace, W. S. Harpole, A. S. MacDougall, and E. W. Seabloom. 2017. A decade of insights into grassland ecosystem responses to global environmental change. Nature Ecology \& Evolution 1:1-7.

Collins, S. L., and S. M. Glenn. 1991. Importance of spatial and temporal dynamics in species regional abundance and distribution. Ecology 72:654-664.

Collins, S. L., K. N. Suding, E. E. Cleland, M. Batty, S. C. Pennings, K. L. Gross, J. B. Grace, L. Gough, J. E. Fargione, and C. M. Clark. 2008. Rank clocks and plant community dynamics. Ecology 89:3534-3541.

Coyle, J. R., A. H. Hurlbert, and E. P. White. 2013. Opposing mechanisms drive richness patterns of core and transient bird species. American Naturalist 181:E83-E90.

Dawson, W., M. Fischer, and M. van Kleunen. 2012. Common and rare plant species respond differently to fertilisation and competition, whether they are alien or native. Ecology Letters 15:873-880.

Dolan, J. R., M. E. Ritchie, A. Tunin-Ley, and M. Pizay. 2009. Dynamics of core and occasional species in the marine plankton: tintinnid ciliates in the north-west Mediterranean Sea. Journal of Biogeography 36:887-895.

Duwyn, A., and A. S. MacDougall. 2015. When anthropogenicrelated disturbances overwhelm demographic persistence mechanisms. Journal of Ecology 103:761-768.

Franklin, J., J. M. Serra-Diaz, A. D. Syphard, and H. M. Regan. 2016. Global change and terrestrial plant community dynamics. Proceedings of the National Academy of Sciences USA 113:3725-3734.

Gaston, K. J. 1994. What is rarity? In Rarity. Springer, Dordrecht, The Netherlands.

Gleason, H. A. 1926. The individualistic concept of the plant association. Bulletin of the Torrey Botanical Club 53:7-26.

Griggs, R. F. 1940. The ecology of rare plants. Bulletin of the Torrey Botanical Club 67:575-594.

Grime, J. 1977. Evidence for the existence of three primary strategies in plants and its relevance to ecological and evolutionary theory. American Naturalist 111:1169-1194.

Grime, J. P. 1998. Benefits of plant diversity to ecosystems: immediate, filter and founder effects. Journal of Ecology 86:902-910

Hanski, I. 1982. Dynamics of regional distribution: the core and satellite species hypothesis. Oikos 38:210-221.

Harpole, W. S., et al. 2016. Addition of multiple limiting resources reduces grassland diversity. Nature 537:93-96.

Hautier, Y., et al. 2014. Eutrophication weakens stabilizing effects of diversity in natural grasslands. Nature 508:521-525.

Hautier, Y., et al. 2020. General destabilizing effects of eutrophication on grassland productivity at multiple spatial scales. Nature Communications 11:1-9.

Hautier, Y., D. Tilman, F. Isbell, E. W. Seabloom, E. T. Borer, and P. B. Reich. 2015. Anthropogenic environmental changes affect ecosystem stability via biodiversity. Science 348:336340.

Hillebrand, H., et al. 2017. Biodiversity change is uncoupled from species richness trends: Consequences for conservation and monitoring. Journal of Applied Ecology 55:169-184. 
Hodapp, D., et al. 2018. Spatial heterogeneity in species composition constrains plant community responses to herbivory and fertilisation. Ecology Letters 21:1364-1371.

Komatsu, K. J., et al. 2019. Global change effects on plant communities are magnified by time and the number of global change factors imposed. Proceedings of the National Academy of Sciences USA 116:17867-17873.

Kuznetsova, A., P. B. Brockhoff, and R. H. B. Christensen. 2017. lmerTest package: tests in linear mixed effects models. Journal of Statistical Software 82:1-26.

Lande, R. 1993. Risks of population extinction from demographic and environmental stochasticity and random catastrophes. American Naturalist 142:911-927.

Langley, J. A., et al. 2018. Ambient changes exceed treatment effects on plant species abundance in global change experiments. Global Change Biology 24:5668-5679.

Lenth, R., H. Singmann, J. Love, P. Buerkner, and M. Herve. 2018. Emmeans: estimated marginal means, aka least-squares means. R package version 1:3. https://CRAN.R-project.org/ package $=$ emmeans

Levin, S. A., D. Cohen, and A. Hastings. 1984. Dispersal strategies in patchy environments. Theoretical Population Biology 26:165-191

Lind, E. M., et al. 2013. Life-history constraints in grassland plant species: a growth-defence trade-off is the norm. Ecology Letters 16:513-521.

Magurran, A. E., and P. A. Henderson. 2003. Explaining the excess of rare species in natural species abundance distributions. Nature 422:714-716.

Manne, L. L., and S. L. Pimm. 2001. Beyond eight forms of rarity: which species are threatened and which will be next? Animal Conservation 4:221-229.

Matthies, D., I. Bräuer, W. Maibom, and T. Tscharntke. 2004. Population size and the risk of local extinction: empirical evidence from rare plants. Oikos 105:481-488.

Mouillot, D., N. A. J. Graham, S. Villéger, N. W. H. Mason, and D. R. Bellwood. 2013. A functional approach reveals community responses to disturbances. Trends in Ecology \& Evolution 28:167-177.

Nakagawa, S., P. C. D. Johnson, and H. Schielzeth. 2017. The coefficient of determination $\mathrm{R}^{2}$ and intra-class correlation coefficient from generalized linear mixed-effects models revisited and expanded. Journal of the Royal Society Interface 14:20170213.

R Core Team. 2020. R: a language and environment for statistical computing. Version 4.0.2. R Core Team, Vienna, Austria. https://www.R-project.org

Rabinowitz, D. 1981. Seven forms of rarity. In The biological aspects of rare plant conservation. Springer, Somerset, New Jersey, USA.

Rabinowitz, D., J. K. Rapp, and P. M. Dixon. 1984. Competitive abilities of sparse grass species: means of persistence or cause of abundance. Ecology 65:1144-1154.
Reich, P. B., D. S. Ellsworth, M. B. Walters, J. M. Vose, C. Gresham, J. C. Volin, and W. D. Bowman. 1999. Generality of leaf trait relationships: a test across six biomes. Ecology 80:1955-1969.

Ripley, B., and W. Venables. 2016. Package 'nnet': feed-forward neural networks and multinomial log-linear models. CRAN R Core Team. https://www.stats.ox.ac.uk/pub/MASS4/

Roxburgh, S. H., K. Shea, and J. B. Wilson. 2004. The intermediate disturbance hypothesis: Patch dynamics and mechanisms of species coexistence. Ecology 85:359-371.

Seabloom, E. W., et al. 2020. Increasing effects of chronic nutrient enrichment on plant diversity loss and ecosystem productivity over time. Ecology 102:3218.

Seabloom, E. W., E. T. Borer, Y. M. Buckley, E. E. Cleland, K. F. Davies, J. Firn, W. S. Harpole, Y. Hautier, E. M. Lind, and A. S. MacDougall. 2015. Plant species' origin predicts dominance and response to nutrient enrichment and herbivores in global grasslands. Nature Communications 6:7710.

Shade, A., and J. A. Gilbert. 2015. Temporal patterns of rarity provide a more complete view of microbial diversity. Trends in Microbiology 23:335-340.

Shade, A., S. E. Jones, J. G. Caporaso, J. Handelsman, R. Knight, N. Fierer, and J. A. Gilbert. 2014. Conditionally rare taxa disproportionately contribute to temporal changes in microbial diversity. mBio 5:e01371-14.

Shoemaker, L. G., et al. 2020. Integrating the underlying structure of stochasticity into community ecology. Ecology 101:117.

Steffen, W., et al. 2015. Planetary boundaries: Guiding human development on a changing planet. Science 347:1259855.

Suding, K. N., S. L. Collins, L. Gough, C. Clark, E. E. Cleland, K. L. Gross, D. G. Milchunas, and S. Pennings. 2005. Functional- and abundance-based mechanisms explain diversity loss due to $\mathrm{N}$ fertilization. Proceedings of the National Academy of Sciences USA 102:4387-4392.

Supp, S. R., D. N. Koons, and S. K. M. Ernest. 2015. Using life history trade-offs to understand core-transient structuring of a small mammal community. Ecosphere 6:1-15.

Tilman, D. 1993. Species richness of experimental productivity gradients: how important is colonization limitation? Ecology 74:2179-2191.

Ulrich, W., and M. Ollik. 2004. Frequent and occasional species and the shape of relative-abundance distributions. Diversity and Distributions 10:263-269.

Umaña, M. N., C. Zhang, M. Cao, L. Lin, and N. G. Swenson. 2017. A core-transient framework for trait-based community ecology: an example from a tropical tree seedling community. Ecology Letters 20:619-628.

Wilfahrt, P. A., et al. 2021. Species persistence and mean rank abundance in global Nutrient Network plots from 2007-2019 ver 1. Environmental Data Initiative. https://doi.org/10.6073/ pasta/e21f6c3cd2615b9295f9e493f6c04591

\section{SUPPORTING INFORMATION}

Additional supporting information may be found in the online version of this article at http://onlinelibrary.wiley.com/doi/ 10.1002/ecy.3504/suppinfo

\section{Open Research}

Data and R code (Wilfahrt et al. 2021) used for all analyses in this paper are available on the EDI data portal: https://doi.org/10. 6073/pasta/e21f6c3cd2615b9295f9e493f6c04591. 